TI 2015-080/II

Tinbergen Institute Discussion Paper

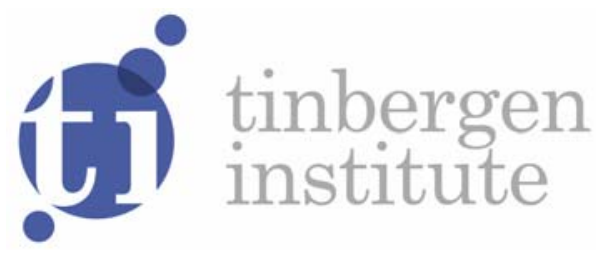

\title{
Note on 'Competition in Two-sided Markets'
}

\author{
Yuyu Zeng \\ Harold Houba \\ Gerard van der Laan
}

Faculty of Economics and Business Administration, VU University Amsterdam, and Tinbergen Institute, the Netherlands. 
Tinbergen Institute is the graduate school and research institute in economics of Erasmus University Rotterdam, the University of Amsterdam and VU University Amsterdam.

More TI discussion papers can be downloaded at http://www.tinbergen.nl

Tinbergen Institute has two locations:

Tinbergen Institute Amsterdam

Gustav Mahlerplein 117

1082 MS Amsterdam

The Netherlands

Tel.: +31(0)205251600

Tinbergen Institute Rotterdam

Burg. Oudlaan 50

3062 PA Rotterdam

The Netherlands

Tel.: +31(0)10 4088900

Fax: $+31(0) 104089031$

Duisenberg school of finance is a collaboration of the Dutch financial sector and universities, with the ambition to support innovative research and offer top quality academic education in core areas of finance.

DSF research papers can be downloaded at: http://www.dsf.nl/

Duisenberg school of finance

Gustav Mahlerplein 117

1082 MS Amsterdam

The Netherlands

Tel.: +31(0)20 5258579 


\title{
Note on 'Competition in Two-sided Markets'*
}

\author{
Yuyu Zeng ${ }^{\dagger}$ \\ VU University Amsterdam \\ and Tinbergen Institute
}

\author{
Harold Houba \\ VU University Amsterdam \\ and Tinbergen Institute
}

\author{
Gerard van der $\operatorname{Laan}^{\S}$ \\ VU University Amsterdam \\ and Tinbergen Institute
}

\begin{abstract}
We extend the models in "Competition in two-sided markets" of Armstrong (2006, Rand Journal of Economics) by adding within-group externalities. In the monopoly and duopoly cases, positive within-group externalities reduce the price of the own group. Negative externalities have an opposite price effect. In the case of a competitive bottleneck, we show by examples that within a certain range of parameter values, a novel phenomenon arises that the platform attracts more agents from one of the groups compared with the social optimum.
\end{abstract}

Keywords: Competition economics, two-sided market

JEL codes: D4, L4

*This research is financially supported by the Netherlands Organization for Scientific Research NWO grant 022.003.035. The authors thank Adriaan Soetevent, Mark Armstrong, Evgenia Motchenkova and Jeroen Hinloopen for valuable suggestions.

${ }^{\dagger}$ Department of Econometrics, VU University Amsterdam, De Boelelaan 1105, 1081 HV Amsterdam, the Netherlands. Email: y.zeng@vu.nl.

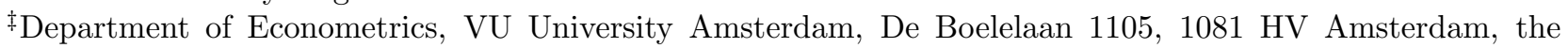
Netherlands. Email: harold.houba@vu.nl.

§Department of Econometrics, VU University Amsterdam, De Boelelaan 1105, 1081 HV Amsterdam, the Netherlands. Email: g.vander.laan@vu.nl. 


\section{Introduction}

Many markets with network externalities, if not most, are two-sided. Following Rochet and Tirole (2006) we can say that "Two-sided (or, more generally, multi-sided) markets are roughly defined as markets in which one or several platforms enable interactions between end-users and try to get the two (or multiple) sides 'on board' by appropriately charging each side. That is, platforms court each side while attempting to make, or at least not lose, money overall." For instance, credit cards companies such as Visa, Mastercard must capture both cardholders and merchants. A wide acceptance of merchants will facilitate the cardholders. More cardholders will reversely stimulate wider acceptance of credit card among merchants. To succeed, platforms in industries such as software, portals, media, payment systems and internet shops must get both sides of the market on board. A special feature of Facebook, YouTube, WhatsApp and other social media is that users experience a higher value if more other users, their peers, use it. This is an example of positive within-group externalities. In contrast, sellers of almost identical goods and bidders for these goods on internet auction eBay experience more competition if more users of their own side participate in the auction. This is an example of negative within-group externalities. The investigation of two-sided markets with within-group externalities is the main topic of this note.

Although Rochet and Tirole (2006) define "a two-sided market as one in which the volume of transactions between end-users depends on the structure and not only on the overall level of fees charged by the platform", the literature on two-sided market mainly focuses on the pricing strategy used by the platforms. Armstrong (2006) investigates the pricing strategy for a monopoly platform and the case of two platforms competing for users. In the latter case, he differentiates between "single-homing" and "multi-homing". An user is "single-homing" when he uses only one platform and "multi-homing" when he chooses several platforms. For the case of two competing platforms, Armstrong (2006) investigates the following two situations: (i) both groups are "single-homing", (ii) one group is "single-homing" and the other is "multi-homing". The case of multiple platforms is also considered in van Cayseele and Reynaerts (2011), where one side of the market has to use all platforms (thus "multi-homing") for either cultural or legal reasons and the other side is "single-homing". They show that in presence of independent platforms, the platform and industry profits are lower compared with the situation of joint ownership of platforms.

In the literature on two-sided markets, most of the focus is on cross-group externalities and little attention has been paid to within-group externalities. Although the key part is the pushup mechanism and price adjustment due to cross-group externalities, within-group externalities should also be taken into consideration, since their interactive effects may influence the final result. In the setting of one-sided markets, typically negative within-group externalities exist. For instance, more cars on a highway increase the possibility of traffic jams and therefore the 
expected traveling time. For a two-sided market we already discussed examples of pure positive and negative within-group externalities. Intermediate cases may also exist. For example, when a heterosexual club has only a few females, an increase in females will attract more males and this in turn makes it more attractive for females. So, at low numbers there are positive withingroup externalities. However it might also be that when the number of females increases beyond some number, the competition between females becomes so tense that it wipes out the positive externalities of attracting more males and for high numbers the within-group externalities might become negative. In this context, it might be appropriate to use a single-peaked form, say quadratic, of within-group externalities. To focus our analysis, we mainly focus on such singlepeaked externalities. The other mentioned examples can be derived in a straightforward manner.

In this note we will investigate how within-group externalities affect the results obtained for the three different models considered by Armstrong (2006). In Section 2 we analyze the effect of within-group externalities for the pricing strategy of a monopoly platform. In Section 3 we consider the case of two competing platforms with "single-homing" platforms on both sides. Finally, in Section 4 we analyze within-group externalities for the competitive bottleneck, i.e., one side is "single-homing" and the other side is "multi-homing".

\section{Monopoly platforms}

In this section we analyze the effects of within-group externalities for a monopoly platform as discussed in Section 3 of Armstrong (2006). For instance, buyers and sellers in a small town can only use one shopping mall to transact commodities. With a rich variety of sellers, consumers can get easy access to what they are searching for, while retailers profit from an increasing number of consumers. Hence, there exist positive cross-group externalities between the two sides of the market. However, we also observe that more retailers in the mall will intensify the competition among similar product providers, so there may exist within-group externalities among the sellers. In this section, we allow for within-group externalities for both groups.

Following Armstrong (2006), we refer to the two sides of the market as group 1 and group 2 respectively. We index these groups by $i, j=1,2$ with $j \neq i$. In this model, utilities are the main decision variables instead of prices. The utility for agents of group $i, i=1,2$, provided by the platform is denoted as $u_{i}$, the entrance fee or price for agents of group $i$ as $p_{i}$, and the number of group $i$ agents attracted by the platform as $n_{i}$. There is an unlimited "pool" of agents on both sides and agents have heterogenous outside options or reservation values drawing from a certain distribution. Given the utility provided by the platform, the number of agents on the platform is given by

$$
n_{i}=\phi_{i}\left(u_{i}\right), \quad i=1,2,
$$


where $\phi_{i}$ is some exogenous function with the property that $\phi_{i}^{\prime}>0$. This means that a higher utility attracts more agents. Cross-group externalities are positive and an agent in group $i$ gets a fractional utility $\alpha_{i}>0$ from the number of agents of group $j$ on the platform. Novel are within-group externalities associated with the number of agents of his group. For an agent of group $i$ these externalities are given by a nonnegative function $g_{i}: \mathbb{R}_{+} \rightarrow \mathbb{R}_{+}$, satisfying $g_{i}(0)=0$ and $g_{i}{ }^{\prime}(0) \geq 0$. Further we assume that for sufficiently large $\bar{x}_{i}>0$, possibly $\bar{x}_{i}$ is infinite, $g_{i}$ is differentiable on the interval $\left[0, \bar{x}_{i}\right]$ and $g_{i}\left(x_{i}\right)=0$ when $x_{i}>\bar{x}_{i}$. The utility for an agent reads,

$$
u_{i}=\alpha_{i} n_{j}+g_{i}\left(n_{i}\right)-p_{i}, \quad i=1,2 .
$$

It could be that $p_{i}<0$, for instance when the manager of a mall in a small town wants to stimulate the customers to visit the mall. The profit realized by the platform is given by

$$
\pi=n_{1}\left(p_{1}-f_{1}\right)+n_{2}\left(p_{2}-f_{2}\right)
$$

where $f_{i}$ is some constant marginal costs for the platform to serve an agent of group $i$. Solving $p_{i}, i=1,2$, from equation (2) we obtain

$$
p_{i}=\alpha_{i} n_{j}+g_{i}\left(n_{i}\right)-u_{i}
$$

and substituting equations (1) and (4) into equation (3) we obtain

$$
\pi\left(u_{1}, u_{2}\right)=\sum_{i=1,2}\left(\phi_{i}\left(u_{i}\right)\left(\alpha_{i} \phi_{j}\left(u_{j}\right)+g_{i}\left(\phi_{i}\left(u_{i}\right)\right)-u_{i}-f_{i}\right)\right)
$$

Taking first-order conditions with respect to $u_{i}, i=1,2$, yields

$$
\phi_{i}^{\prime}\left(u_{i}\right)\left[\alpha_{i} \phi_{j}\left(u_{j}\right)+g_{i}\left(\phi_{i}\left(u_{i}\right)\right)-u_{i}-f_{i}\right]+\phi_{i}\left(u_{i}\right)\left[g_{i}^{\prime}\left(\phi_{i}\left(u_{i}\right)\right) \phi_{i}^{\prime}\left(u_{i}\right)-1\right]+\phi_{j}\left(u_{j}\right) \alpha_{j} \phi_{i}^{\prime}\left(u_{i}\right)=0 .
$$

For the monopoly platform, the optimal utility levels $u_{i}$ provided by the platform are obtained by solving the two equations (5) for $i=1,2$. Then the numbers of agents $n_{i}$ are obtained by substituting $u_{i}$ in (1) and the prices $p_{i}$ from substituting $u_{i}$ and $n_{i}$ in equation (4).

When substituting (2) back into (5) we obtain

$$
p_{i}=f_{i}-\alpha_{j} \phi_{j}\left(u_{j}\right)-\phi_{i}\left(u_{i}\right) g_{i}^{\prime}\left(\phi_{i}\left(u_{i}\right)\right)+\frac{\phi_{i}\left(u_{i}\right)}{\phi_{i}^{\prime}\left(u_{i}\right)}, i=1,2
$$

Compared with equation (3) of Armstrong (2006) and noticing that $\phi_{j}\left(u_{j}\right)=n_{j}$, equation (6) has the additional term $-\phi_{i}\left(u_{i}\right) g_{i}^{\prime}\left(\phi_{i}\left(u_{i}\right)\right)$. So, in addition to reducing the price for a group $i$ agent by his contribution to group $j$ due to positive cross-group externalities, reflected by the second term $\alpha_{j} \phi_{j}\left(u_{j}\right)$, the price for a group $i$ agent is also adjusted for his within-group externalities given 
by the $-\phi_{i}\left(u_{i}\right) g_{i}^{\prime}\left(\phi_{i}\left(u_{i}\right)\right)$. If $g_{i}^{\prime}>0$, then within-group externalities are positive and joining the platform benefits other members of his group and the price should be reduced by a downward factor that is the product of the number of group $i$ agents and his marginal contribution to his own group. If $g_{i}^{\prime}<0$, then within-group externalities are negative and the participation of another agent $i$ decreases the utility of agents of his own group and hence the platform should charge him a higher price.

Note that the optimal utility levels $u_{1}$ and $u_{2}$ are second-best in the sense that they maximize the monopoly profit of the platform, ignoring the benefits of the customers. We therefore also consider social welfare and maximize the sum of the platform profit and the consumer surplus (CS hereafter),

$$
W\left(u_{1}, u_{2}\right)=\pi\left(u_{1}, u_{2}\right)+C S_{1}\left(u_{1}\right)+C S_{2}\left(u_{2}\right)
$$

where $C S_{i}\left(u_{i}\right)=\int_{0}^{u_{i}} \phi_{i}(z) d z, i=1,2$. Noticing that $C S_{i}^{\prime}\left(u_{i}\right)=\phi_{i}\left(u_{i}\right)$, the socially-optimal price bundle $\left\{p_{1}, p_{2}\right\}$ should satisfy the first-order condition

$$
p_{i}=f_{i}-\alpha_{j} \phi_{j}\left(u_{j}\right)-\phi_{i}\left(u_{i}\right) g_{i}^{\prime}\left(\phi_{i}\left(u_{i}\right)\right), i=1,2
$$

The social optimal price for group $i$ equals the cost of serving a group $i$ agent, adjusted by the external benefit that an additional group $i$ agent brings to the group $j$ agents on the platform and the within-group externalities he imposes on his own group members. Compared with the second-best result, the social optimal price level is reduced by $\frac{\phi_{i}\left(u_{i}\right)}{\phi_{i}^{\prime}\left(u_{i}\right)}>0$, which confirms common intuition in monopoly cases. We summarize the results in the next proposition.

Proposition 2.1. The price $p_{i}$ offered by the platform to an agent of group $i$ is reduced by cross-group externalities $\alpha_{j} \phi_{j}\left(u_{j}\right)$ and adjusted by within-group externalities $-\phi_{i}\left(u_{i}\right) g_{i}^{\prime}\left(\phi_{i}\left(u_{i}\right)\right)$. Depending on group $i$ agent's marginal contribution to his own group, the latter is a price reduction in case of positive within-group externalities, and a price increase otherwise. Finally, the monopoly platform charges a higher price for both group agents compared with the social optimum.

Proposition 2.1 extends the results reported in Armstrong (2006) by adding within-group externalities. When taking $g_{i}\left(x_{i}\right)=0$ for every $x_{i} \geq 0$, equation (6) reduces to equation (3) of Armstrong (2006).

\section{Duopoly platforms with single-homing agents}

In this section, we generalize the monopoly model to the duopoly case of two platforms competing for two groups of agents that are all "single-homing" in the presence of within-group externalities. 
We modify the procedure for the monopoly case to demonstrate how it can be easily adapted to solve the duopoly case in terms of equilibrium utilities. Our duopoly model extends the model discussed in Section 4 of Armstrong (2006) in two ways that we allow for general functional forms and within-group externalities. We conclude this section with an example in which we add within-group externalities to the model discussed in Section 4 of Armstrong (2006).

\subsection{The duopoly model}

There are two platforms that we index as $h, k=A, B$ and we distinguish platform $h$ from platform $k$, i.e., $h \neq k$. As before, the two groups of agents are indexed as $i, j=1,2$ and $j \neq i$. Platform $h, h,=A, B$ offers agents of group $i, i=1,2$, a utility of $u_{i}^{h}$, an entrance fee of $p_{i}^{h}$ and the platform attracts $n_{i}^{h}$ of group $i$ agents. Generalizing both our monopoly model and Armstrong (2006), who considers the Hotelling specification of our example below, we consider the general situation that agents are attracted to the platforms by the utilities they receive. Given the utilities $\left(u_{i}^{h}, u_{i}^{k}\right)$ offered by the platforms to a consumer of group $i$, the number $n_{i}^{h}$ of group $i$ agents attracted by platform $h$ is given by the differentiable function,

$$
n_{i}^{h}=\phi_{i}^{h}\left(u_{i}^{h}, u_{i}^{k}\right)
$$

where $\frac{\partial \phi_{i}^{h}}{\partial u_{i}^{h}}>0$ and $\frac{\partial \phi_{i}^{h}}{\partial u_{i}^{k}}<0$. This captures that the number of agents of any group that uses platform $h$ is increasing in the utility offered by this platform and decreasing in the utility offered by its competitor. Generalizing Armstrong (2006), we also consider the situation that there are not only cross-group externalities, but also within-group externalities for both groups. For an agent of group $i$, these externalities are given by a nonnegative function $g_{i}^{h}: \mathbb{R}_{+} \rightarrow \mathbb{R}_{+}$that has the same properties as the function $g_{i}$ of the monopoly case. The utilities of the agents of group $i$ on platform $h$ are given by

$$
u_{i}^{h}=\alpha_{i} n_{j}^{h}+g_{i}^{h}\left(n_{i}^{h}\right)-p_{i}^{h}
$$

or equivalently we have

$$
p_{i}^{h}=\alpha_{i} n_{j}^{h}+g_{i}^{h}\left(n_{i}^{h}\right)-u_{i}^{h} .
$$

Platform $h$ has constant marginal costs $f_{i}^{h}$ to serve a customer of group $i$. So, the profit for platform $h$ reads

$$
\pi^{h}=\left(p_{1}^{h}-f_{1}^{h}\right) n_{1}^{h}+\left(p_{2}^{h}-f_{2}^{h}\right) n_{2}^{h} .
$$

Similar as for the monopoly case, after substituting equations (7) and (9) into the profit function 
(10) we get

$$
\pi^{h}\left(u_{1}^{h}, u_{2}^{h} ; u_{1}^{k}, u_{2}^{k}\right)=\sum_{i=1,2}\left(\alpha_{i} \phi_{j}^{h}\left(u_{j}^{h}, u_{j}^{k}\right)+g_{i}^{h}\left(\phi_{i}^{h}\left(u_{i}^{h}, u_{i}^{k}\right)\right)-u_{i}^{h}-f_{i}^{h}\right) \phi_{i}^{h}\left(u_{i}^{h}, u_{i}^{k}\right)
$$

Both platforms in the duopoly are profit maximizers and the utilities that they offer are their decision variables. Taking the partial derivatives with respect to all utilities, we obtain the first-order conditions for $h, k=A, B$ and $i, j=1,2$,

$$
\frac{\partial \phi_{i}^{h}}{\partial u_{i}^{h}}\left[\left(\alpha_{1}+\alpha_{2}\right) \phi_{j}^{h}\left(u_{j}^{A}, u_{j}^{B}\right)+g_{i}^{h}\left(\phi_{i}^{h}\left(u_{i}^{A}, u_{i}^{B}\right)\right)-u_{i}^{h}-f_{i}^{h}+\left(g_{i}^{h}\right)^{\prime} \phi_{i}^{h}\left(u_{i}^{A}, u_{i}^{B}\right)\right]-\phi_{i}^{h}\left(u_{i}^{A}, u_{i}^{B}\right)=0
$$

The optimal utility levels $u_{i}^{h}$ provided by both platforms are obtained by solving the system of equations (11). Then, the numbers of agents and the prices in the duopoly equilibrium are obtained by substituting the optimal utilities equations (7) and (9). If we substitute equation (8) back into expression (11), we obtain

$$
p_{i}^{h}=f_{i}^{h}-\alpha_{j} \phi_{j}^{h}\left(u_{j}^{A}, u_{j}^{B}\right)-\left(g_{i}^{h}\right)^{\prime} \phi_{i}^{h}\left(u_{i}^{A}, u_{i}^{B}\right)+\left(\partial \phi_{i}^{h} / \partial u_{i}^{h}\right)^{-1} \phi_{i}^{h}\left(u_{i}^{A}, u_{i}^{B}\right)
$$

This expression for the entrance fee of group $i$ agents on platform $h$ is similar to the monopoly case except that the utilities $u_{i}^{k}$ and $u_{j}^{k}$ of the competing platform enter a platform's price setting. If platform $k$ offers a higher utility to group $j$ agents, then platform $h$ will attract less group $j$ agents and one single group $i$ agent on platform $h$ contributes less to his cross groups due to the fact that there are less group $j$ agents on the platform $h$, therefore the price $p_{i}^{h}$ will increase. The impact of the utility that platform $k$ offers to group $i$ agents enters $p_{i}^{h}$ through the following two terms $-\left(g_{i}^{h}\right)^{\prime} \phi_{i}^{h}\left(u_{i}^{A}, u_{i}^{B}\right)$ and $\left(\partial \phi_{i}^{h} / \partial u_{i}^{h}\right)^{-1} \phi_{i}^{h}\left(u_{i}^{A}, u_{i}^{B}\right)$.

We conclude the above discussion with the following proposition.

Proposition 3.1. The price $p_{i}^{h}$ offered by platform $h$ to an agent of group $i$ is reduced by crossgroup externalities $\alpha_{j} \phi_{j}^{h}\left(u_{j}^{A}, u_{j}^{B}\right)$, adjusted by within-group externalities $-\left(g_{i}^{h}\right)^{\prime} \phi_{i}^{h}\left(u_{i}^{A}, u_{i}^{B}\right)$ and $\left(\partial \phi_{i}^{h} / \partial u_{i}^{h}\right)^{-1} \phi_{i}^{h}\left(u_{i}^{A}, u_{i}^{B}\right)$. Depending on group $i$ agent's marginal contribution to his own group, the influence of within-group externalities is a price reduction in case of positive within-group externalities, and a price increase otherwise.

Note that the duopoly equilibrium utility levels are second-best in the sense that they maximize the profits on each platform, ignoring the benefits of the customers. For duopolies with a fixed group size and platforms that serve the entire market, such as for the Hotelling specification, it is meaningless to discuss social welfare. Since if both platforms have the same constant marginal costs, social welfare is constant. If a platform sets a higher price and loses one customer, this customer does not disappear from the market but instead joins the competing platform. 


\section{$3.2 \quad$ An example}

In this example, we revisit the model of Section 4 in Armstrong (2006) with the Hotelling specification and extend it by adding within-group externalities.

Suppose that for each group the customers are shared between the two platforms according to the Hotelling specification. Let the size of group $i, i=1,2$, be $k_{i}>0$ and each agent has per-unit transportation costs of $t_{i}>0$. According to the Hotelling specification, the number of group $i$ agents is shared between the platforms according to

$$
n_{i}^{h}=\phi_{i}^{h}\left(u_{i}^{A}, u_{i}^{B}\right)=\left(\frac{1}{2}+\frac{u_{i}^{h}-u_{i}^{k}}{2 t_{i}}\right) k_{i} .
$$

Note that $n_{i}^{h}+n_{i}^{k}=k_{i}$, so all group $i$ agents are served. Suppose that the pattern of withingroup externalities is the same on both platforms such that $g_{i}^{h}\left(n_{i}^{h}\right)=-a_{i}\left(n_{i}^{h}\right)^{2}+2 b_{i} n_{i}^{h}$, where $a_{i}, b_{i}>0$ allows that groups may have different values for $a_{i}$ and $b_{i}$. So, before the peak $\frac{b_{i}}{a_{i}}$ the within-group externalities are positive and beyond this peak within-group externalities are negative. The first-order conditions given in equation (11) become for $h=A, B$ and $i=1,2$,

$$
\begin{aligned}
& \frac{1}{2 t_{i}}\left[\left(\alpha_{1}+\alpha_{2}\right)\left(\frac{1}{2}+\frac{u_{j}^{A}-u_{j}^{B}}{2 t_{j}}\right) k_{j}-a_{i}\left(\frac{1}{2}+\frac{u_{i}^{A}-u_{i}^{B}}{2 t_{i}}\right)^{2} k_{i}^{2}+2 b_{i}\left(\frac{1}{2}+\frac{u_{i}^{A}-u_{i}^{B}}{2 t_{i}}\right)\right] \\
&+\frac{1}{2 t_{i}}\left[-u_{i}^{A}-f_{i}^{A}-2 a_{i}\left(\left(\frac{1}{2}+\frac{u_{i}^{A}-u_{i}^{B}}{2 t_{i}}\right) k_{i}-\frac{b_{i}}{a_{i}}\right)\left(\frac{1}{2}+\frac{u_{i}^{A}-u_{i}^{B}}{2 t_{i}}\right)\right]-\left(\frac{1}{2}+\frac{u_{i}^{A}-u_{i}^{B}}{2 t_{i}}\right)=0 .
\end{aligned}
$$

As a special case, we consider the situation that two groups have the same group size and we normalize this size to one, thus $k_{1}=k_{2}=1$, and that the two platforms have the same constant marginal costs, i.e., $f_{i}^{h}=f_{i}^{k}=f_{i}$. Consider the symmetric equilibrium with $u_{i}^{h}=u_{i}^{k}=u_{i}$. Then it follows that $n_{i}^{h}=n_{i}^{k}=\frac{1}{2}$. From equation (14) we obtain

$$
u_{i}=\frac{1}{2} \alpha_{i}+\frac{1}{2} \alpha_{j}+g_{i}\left(\frac{1}{2}\right)-f_{i}-\left(\frac{1}{2} a_{i}-b_{i}\right)-t_{i} .
$$

It follows that ${ }^{1}$

$$
p_{i}=f_{i}+t_{i}+\left(\frac{1}{2} a_{i}-b_{i}\right)-\frac{1}{2} \alpha_{j} .
$$

\footnotetext{
${ }^{1}$ Expression (7) in Armstrong (2006), where $a_{1}=b_{1}=0$, states $p_{1}=f_{1}+t_{1}-\alpha_{2}$. This result is obtained if utilities are substituted out before taking the first-order conditions, which we think is the incorrect order of deriving the Nash equilibrium in this model. Our results are derived under the opposite order.
} 
The equilibrium price in the duopoly is adjusted by both the cross-group externalities and the within-group externalities. In response to the positive cross-group externalities, the price of an group $i$ agent is adjusted downward proportional to his marginal contribution, $\alpha_{j}$, to the cross-group agents. For instance, the price for group 1 agents is pushed downward by $\frac{1}{2} \alpha_{2}$. An explanation for the fraction $\frac{1}{2}$ is that in the Hotelling specification, if the price charged on one group by the platform decreases by one unit, the number of agents only increases by a half unit. Moreover, in equilibrium only half of the interval of each group is captured by each platform. So the price charged on group 1 should be lowered by his contribution to only half of the total group 2 agents. The equilibrium price is also determined by his own within-group externalities $-\left(g_{i}^{h}\right)^{\prime}\left(\frac{1}{2}\right)=a_{i}-2 b_{i}$ according to (12). The sign of these externalities depends upon the peak $\frac{b_{i}}{a_{i}}$. For $\frac{b_{i}}{a_{i}}<\frac{1}{2}$, within-group externalities are locally negative and the price is adjusted upward. For $\frac{b_{i}}{a_{i}}>\frac{1}{2}$, within-group externalities are locally positive and the price is adjusted downward.

For the special case of linear within-group externalities in both groups, i.e., $a_{1}=a_{2}=0$, the analysis above remains valid and the only difference is that $a_{i}$ drops out of the expression for $p_{i}$. Because the coefficient $b_{i}$ is positive, the within-group externalities of group $i$ are always positive and each platform lowers its equilibrium price to this group by $b_{i}$ to attract group $i$ agents.

\section{Competitive bottleneck platforms}

In this section we analyze within-group externalities for the competitive bottleneck model discussed in Section 5 of Armstrong (2006). In this model one of the groups is still "single-homing", while the other group is "multi-homing".

In a setting of competing mobile telecommunications networks, Armstrong (2006) differentiates between mobile subscribers and people on fixed telephony networks who wish to call mobile subscribers. In general, each subscriber ("single-homing") uses only one mobile network and pays the subscription fees and those people on the fixed telephony network ("multi-homing") should have access to all mobile networks and pay termination charges. In equilibrium, mobile networks will charge a low subscription fee and a high call termination fee. The mobile networks pass on the profits from high call termination charges to subsidize the mobile subscribers. Putting it differently, the equilibrium call termination charge is chosen to maximize the profits of mobile network and subscribers while ignoring the benefits of people on the fixed telephony network. The feature that the "single-homing" side is treated well and the interests of "multi-homing" side are ignored in equilibrium is present in a competitive bottleneck setting.

According to Proposition 4 of Armstrong (2006), there are too few "multi-homing" agents served on the platform given the distribution of "single-homing" agents on the platform. In general this implies a market failure. It does not make sense to explain the market failure in terms of competition effects. The position each platform holds is different from two sides. 
From the "single-homing" side, the platform faces a more or less competitive market. From the "multi-homing" side, it holds a local monopoly. The profits the platform collects from the "multi-homing" side might pass on to the competitive side, i.e., the "single-homing" agents on the platform. Armstrong further argues that the market failure is a special feature of this competitive bottleneck, i.e., the "single-homing" side is treated well and the "multi-homing" side is priced aggressively by the platform. In the whole analysis of Armstrong (2006), he ignores the effects of within-group externalities. In the following analysis, we add two forms of within-group externalities.

We focus on the following example in our setting. One customer might use only one airline reservation system to book air tickets. The reason might be that he got good services from previous experiences and is very satisfied with this airline reservation system, therefore he refrains from searching other airline reservation options. So on this airline reservation system, there is a fixed number of market shares of customers in this example. Under this scenario, airline companies are "forced" to deal with all airline reservation systems (i.e., platforms) in order to gain access to as many customers as possible. Moreover, an airline company's decision to join one reservation system is independent from his decision to join another reservation system. In this setup, we model customers as group 1 agents and airline companies as group 2 agents. Note that in this setup, there is no competition between platforms to attract group 2 agents. This differs from the previous section, where group 2 agents are also shared by the two platforms. This can be modeled, by slightly modifying (7) to specify $n_{2}^{h}=\phi_{2}^{h}\left(u_{2}^{h}\right)$, where $\frac{\partial \phi_{2}^{h}}{\partial u_{2}^{h}}>0$ as before. This specification can be seen as the boundary case $\frac{\partial \phi_{2}^{h}}{\partial u_{2}^{k}}=0$ of the model in Section 3. Again, this captures that the share of agents of group 2 that uses platform $h$ is increasing in the utility offered by this platform and it is independent of the utility offered by its competitor.

We consider within-group externalities only among "multi-homing" group 2 agents and not among "single-homing" group 1 agents, i.e., $g_{1}^{h}\left(n_{1}^{h}\right)=0$. For notational convenience, we write $g_{2}\left(n_{2}^{h}\right)$ for $g_{2}^{h}\left(n_{2}^{h}\right)$. What differs from Section 3, is that we perform the analysis in terms of the number of agents attracted to the platforms, where we mainly focus on the "multi-homing" agents. For computational convenience, we further specify that the number of group 2 agents attracted by platform $h$, given that $n_{1}^{h}$ group 1 agents are present, is

$$
n_{2}^{h}=\alpha_{2} n_{1}^{h}-p_{2}^{h}+g_{2}\left(n_{2}^{h}\right)
$$

where $g_{2}\left(n_{2}^{h}\right)$ captures the within group externalities among group 2 agents. We assume $g_{2}(0)=0$.

Because (9) and (10) still hold, we can express the profit of platform $h$ in terms of the number of agents it attracts. The profit function for the platform $h$ reads,

$$
\pi^{h}=n_{1}^{h}\left(\alpha_{1} n_{2}^{h}-u_{1}^{h}\right)+n_{2}^{h}\left(\alpha_{2} n_{1}^{h}-n_{2}^{h}+g_{2}\left(n_{2}^{h}\right)\right)-\left(n_{1}^{h} f_{1}^{h}+n_{2}^{h} f_{2}^{h}\right) .
$$


Because the platforms do not compete for group 1 agents, it is without much loss of generality to write that platform $h$ offers group 1 agents their equilibrium utility $\hat{u}_{1}^{h}$ and, doing so, attracts the equilibrium number $\hat{n}_{1}^{h}$ of group 1 agents. In order to achieve this, platform $h$ needs to adjust its price $p_{1}^{h}$ to the number of group 2 agents it attracts, which means that $p_{1}^{h}=\alpha_{1} n_{2}^{h}-\hat{u}_{1}^{h}$ similar as in previous sections.

Given $\hat{n}_{1}^{h}$, platform $h$ chooses $n_{2}^{h}$ to maximize

$$
r^{h}\left(n_{2}^{h}\right)=\hat{n}_{1}^{h} \alpha_{1} n_{2}^{h}+n_{2}^{h}\left(\alpha_{2} \hat{n}_{1}^{h}-n_{2}^{h}+g_{2}\left(n_{2}^{h}\right)\right)-\left(\hat{n}_{1}^{h} f_{1}^{h}+n_{2}^{h} f_{2}^{h}\right)
$$

and the above expression maximizes the joint interests of group 1 agents (i.e., $\hat{n}_{1}^{h} \hat{u}_{1}^{h}$ ) and the platform while the interests of group 2 agents are ignored. The first-order condition is given by

$$
\frac{\partial r^{h}}{\partial n_{2}^{h}}=\alpha_{1} \hat{n}_{1}^{h}+\alpha_{2} \hat{n}_{1}^{h}-2 n_{2}^{h}+g_{2}\left(n_{2}^{h}\right)+n_{2}^{h} g_{2}^{\prime}\left(n_{2}^{h}\right)-f_{2}^{h}=0 .
$$

Note that in equilibrium $\hat{p}_{2}^{h}=\alpha_{2} \hat{n}_{1}^{h}-\hat{n}_{2}^{h}+g_{2}\left(\hat{n}_{2}^{h}\right)$, where $\hat{n}_{2}^{h}$ solves (17).

Next, we consider social welfare generated by platform $h$. First, note that the aggregate surplus generated by group 2 agents on platform $h$ is given by $\int_{0}^{n_{2}^{h}} p_{2}^{h}(x) d x$, where $p_{2}^{h}(x)$ expresses that the inverse demand function depends upon the number $x$ of group 2 agents on platform $h$. Hence, the aggregate surplus of group 2 agents given $\hat{n}_{1}^{h}$ and $n_{2}^{h}$ on platform $h$ is

$$
\int_{0}^{n_{2}^{h}}\left(\alpha_{2} \hat{n}_{1}^{h}-x+g_{2}(x)\right) d x=\alpha_{2} \hat{n}_{1}^{h} n_{2}^{h}-\frac{1}{2}\left(n_{2}^{h}\right)^{2}+G_{2}\left(n_{2}^{h}\right)-G_{2}(0),
$$

here $G_{2}^{\prime}(x)=g_{2}(x)$. The aggregate surplus of group 1 agents on platform $h$ includes the price the platform collects from group 1 agents and also the utility for group 1 agents on the platform. Given $\hat{n}_{1}^{h}$, the aggregate surplus of group 1 agents on platform $h$ is $\hat{n}_{1}^{h} \alpha_{1} n_{2}^{h}$. Hence, the total surplus on platform $h$ is given by

$$
v^{h}\left(n_{2}^{h}\right)=\hat{n}_{1}^{h} \alpha_{1} n_{2}^{h}+\alpha_{2} \hat{n}_{1}^{h} n_{2}^{h}-\frac{1}{2}\left(n_{2}^{h}\right)^{2}+G_{2}\left(n_{2}^{h}\right)-G_{2}(0)-\left(\hat{n}_{1}^{h} f_{1}^{h}+n_{2}^{h} f_{2}^{h}\right)
$$

The first-order condition for the maximal total surplus is

$$
\frac{\partial v^{h}}{\partial n_{2}^{h}}=\alpha_{1} \hat{n}_{1}^{h}+\alpha_{2} \hat{n}_{1}^{h}-n_{2}^{h}+g_{2}\left(n_{2}^{h}\right)-f_{2}^{h}=0 .
$$

We narrow the welfare analysis to the question whether platform $h$ attracts the socially optimal number of group 2 agents in the duopoly equilibrium. From equations (17) and (19), we obtain

$$
\frac{\partial r^{h}}{\partial n_{2}^{h}}=\frac{\partial v^{h}}{\partial n_{2}^{h}}+n_{2}^{h}\left[g_{2}^{\prime}\left(n_{2}^{h}\right)-1\right]
$$


In the following, we investigate two specific forms of within-group externalities.

\subsection{Linear within-group externalities}

In this subsection we consider the case of linear within group externalities, given by $g_{2}\left(n_{2}^{h}\right)=$ $\beta_{2} n_{2}^{h}$. It follows that $G_{2}\left(n_{2}^{h}\right)=\frac{1}{2} \beta_{2}\left(n_{2}^{h}\right)^{2}+c$, where $c$ is a constant. For $\beta_{2}>0$ the within-group externalities are positive. Note that both (16) and (18) are in quadratic form.

When $g_{2}^{\prime}\left(n_{2}^{h}\right)-1<0$, i.e., $\beta_{2}<1$, the right-hand side of equation (20) is smaller than the left-hand side for any number of $n_{2}^{h}$ and the coefficient of the term of degree 2 in equations (16) and (18) is negative. Let $n_{2}^{h *}$ be the solution for (17) and $\frac{\partial r^{h}}{\partial n_{2}^{h}}\left(n_{2}^{h *}\right)=0$. Then $\frac{\partial v^{h}}{\partial n_{2}^{h}}\left(n_{2}^{h *}\right)>0$. Therefore, the maximizer of (16) is always smaller than the maximizer of (18). There are too few group 2 agents served on the platform compared with the social optimum in case of linear within group externalities when $\beta_{2}<1$. This includes the class of negative within-group externalities $\left(\beta_{2}<0\right)$ and the case with an absence of these externalities $\left(\beta_{2}=0\right)$ in Section 5 of Armstrong (2006).

When $\beta_{2}>1$, the coefficient of the term of degree 2 in equations (16) and (18) is positive. Only the minimizer is attained at the saddle point. In this situation, the maximizers are attained at either 0 or the maximal number of group 2 agents.

\subsection{Quadratic within-group externalities}

We now consider the case of quadratic within-group externalities that are given by $g_{2}\left(n_{2}^{h}\right)=$ $-\left(n_{2}^{h}\right)^{2}+2 b_{2} n_{2}^{h}$, where $b_{2}>0$. The within-group externalities are positive before the peak point $b_{2}$ and negative beyond. We have $G_{2}\left(n_{2}^{h}\right)=-\frac{1}{3}\left(n_{2}^{h}\right)^{3}+b_{2}\left(n_{2}^{h}\right)^{2}+c$, where $c$ is a constant that is irrelevant for the analysis. By substituting $g_{2}\left(n_{2}^{h}\right)$ in (16) and (17) we obtain the platform profit

$$
r^{h}\left(n_{2}^{h}\right)=-\left(n_{2}^{h}\right)^{3}+\left(2 b_{2}-1\right)\left(n_{2}^{h}\right)^{2}+\left(\left(\alpha_{1}+\alpha_{2}\right) \hat{n}_{1}^{h}-f_{2}^{h}\right) n_{2}^{h}-\hat{n}_{1}^{h} f_{1}^{h}
$$

and the first-order condition for profit maximizing

$$
\frac{\partial r^{h}}{\partial n_{2}^{h}}=-3\left(n_{2}^{h}\right)^{2}+2\left(2 b_{2}-1\right) n_{2}^{h}+c_{2}=0
$$




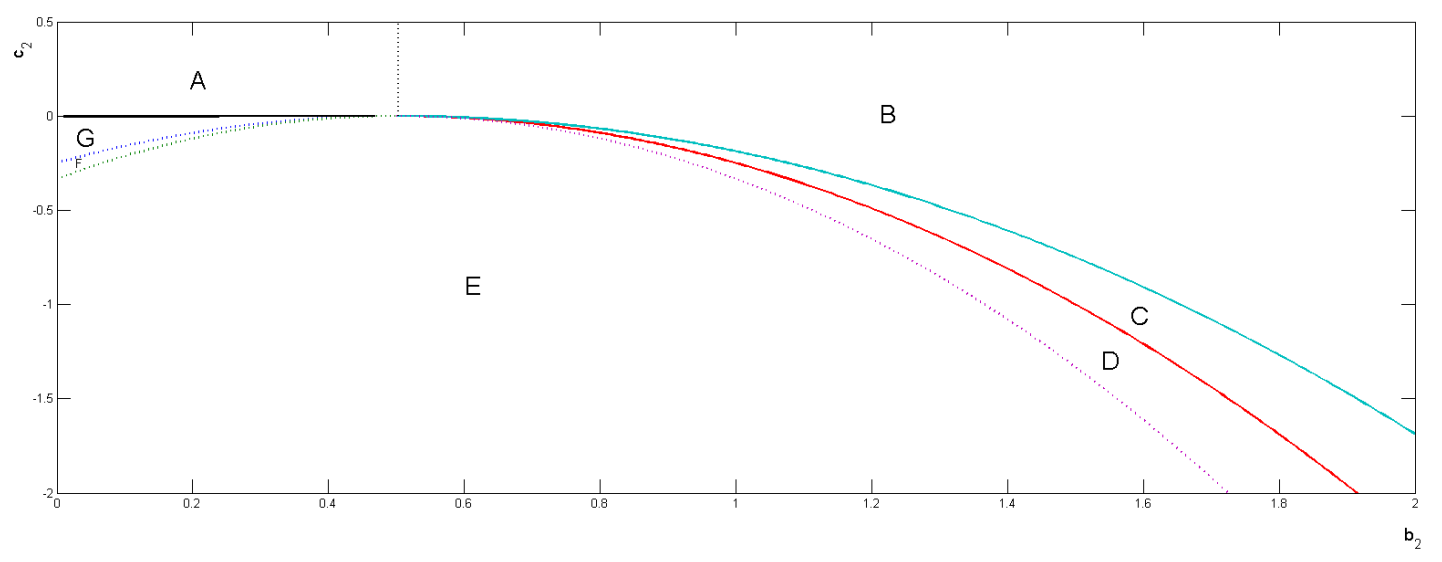

Figure 1: The regions defined in Table 1 in the $\left(b_{2}, c_{2}\right)$ space

where $c_{2}=\left(\alpha_{1}+\alpha_{2}\right) \hat{n}_{1}^{h}-f_{2}^{h}$. Solving for the profit-maximizing number of group 2 agents, we obtain $^{2}$

$$
\hat{n}_{2}^{h}=\frac{\left(2 b_{2}-1\right)+\sqrt{\left(2 b_{2}-1\right)^{2}+3 c_{2}}}{3} .
$$

Similarly, we obtain from (18) and (19), the social welfare

$$
v^{h}\left(n_{2}^{h}\right)=-\frac{1}{3}\left(n_{2}^{h}\right)^{3}+\left(b_{2}-\frac{1}{2}\right)\left(n_{2}^{h}\right)^{2}+\left(\left(\alpha_{1}+\alpha_{2}\right) n_{1}^{h}-f_{2}^{h}\right) n_{2}^{h}-\hat{n}_{1}^{h} f_{1}^{h}
$$

and the first-order condition for welfare maximization

$$
\frac{\partial v^{h}}{\partial n_{2}^{h}}=-\left(n_{2}^{h}\right)^{2}+\left(2 b_{2}-1\right) n_{2}^{h}+c_{2}=0
$$

Then we obtain the welfare-maximizing number of group 2 agents by solving (21),

$$
\hat{\hat{n}}_{2}^{h}=\frac{\left(2 b_{2}-1\right)+\sqrt{\left(2 b_{2}-1\right)^{2}+4 c_{2}}}{2} .
$$

Next, we investigate the question whether the number of group 2 agents on the platform is smaller or bigger compared with the situation of social optimum. In case the platform serves not the same group 2 agents as the social optimum, this implies a market failure. In the following, when we mention the social or platform optimum, we always mean the number of group 2 agents in the platform optimum or social optimum. The ranking of the social and platform optimum depends on $b_{2}$ and $c_{2}$. We summarize the results in Table 1 and plot the corresponding regions

\footnotetext{
${ }^{2}$ The necessary condition for a profit maximum is always satisfied. Alternatively, the profit function is a polynomial of third degree with a negative coefficient in front of the term of third degree. Hence, the optimal number $n_{2}^{h}$ is given by the largest root, which is a local maximum.
} 
Table 1: The characterization of all regions and the optima

\begin{tabular}{|c|c|c|}
\hline Region & range of $b_{2}$ and $c_{2}$ & social and platform optimum \\
\hline$A$ & $0<b_{2}<\frac{1}{2}$ and $c_{2}>0$ & social optimum $>$ platform optimum $>0$ \\
\hline$B$ & $\frac{1}{2}<b_{2}$ and $-\frac{3}{16}\left(2 b_{2}-1\right)^{2}<c_{2}$ & social optimum $>$ platform optimum $>0$ \\
\hline$C$ & $-\frac{\left(2 b_{2}-1\right)^{2}}{4}<c_{2}<-\frac{3}{16}\left(2 b_{2}-1\right)^{2}$ & platform optimum $>$ social optimum $=0$ \\
\hline$D$ & $-\frac{1}{3}\left(2 b_{2}-1\right)^{2} \leq c_{2}<-\frac{\left(2 b_{2}-1\right)^{2}}{4}$ and $b_{2}>\frac{1}{2}$ & Both are 0. \\
\hline$E$ & $c_{2}<-\frac{1}{3}\left(2 b_{2}-1\right)^{2}$ & Both are 0. \\
\hline$F$ & $-\frac{1}{3}\left(2 b_{2}-1\right)^{2}<c_{2} \leq-\frac{1}{4}\left(2 b_{2}-1\right)^{2}$ and $0<b_{2}<\frac{1}{2}$ & Both are 0. \\
\hline$G$ & $0<b_{2}<\frac{1}{2}$ and $-\frac{1}{4}\left(2 b_{2}-1\right)^{2}<c_{2}<0$ & Both are 0. \\
\hline \hline
\end{tabular}

in Figure 1.

In the following, we sketch the calculations. Let $N_{2}$ be the maximal number of group 2 agents that could be served.

In region $\mathrm{A}$ and $\mathrm{B}$, both optimal number of group 2 agents on the platform are positive and $\hat{\hat{n}}_{2}^{h}>\hat{n}_{2}^{h}>0 .^{3}$ There are too few group 2 agents served on the platform compared with the social optimum.

In region $\mathrm{C}$, both optimal number of group 2 agents are positive, but $r^{h}\left(\hat{n}_{2}^{h}\right)>r^{h}(0)$ and $v^{h}\left(\hat{\hat{n}}_{2}^{h}\right)<v^{h}(0)$. Hence, the social optimum yields 0 number of group 2 agents and the platform optimum has a positive number of group 2 agents. This leads to a "novel" phenomenon that too much group 2 agents are served on the platform compared with the social optimum. By taking the advantage of the positive within-group externalities among group 2 agents, the platform could serve more group 2 agents in equilibrium.

In region $\mathrm{D}$, the discriminant for $\hat{\hat{n}}_{2}^{h}$ is negative. No real root for (21) exists. Also, the coefficient of the term of degree 2 for $\frac{\partial v^{h}}{\partial n_{2}^{h}}$ is negative. So, $\frac{\partial v^{h}}{\partial n_{2}^{h}}$ is always negative for any $n_{2}^{h}$ and the social welfare is decreasing in the number of group 2 agents on the platform. The social optimum has 0 group 2 agents. Meanwhile, we have $\hat{n}_{2}^{h}>0$ and $r^{h}\left(\hat{n}_{2}^{h}\right)<r^{h}(0)$. Hence, both the platform and social optimum are achieved at 0 number of group 2 agents. In region E, both the discriminant for $\hat{n}_{2}^{h}$ and $\hat{\hat{n}}_{2}^{h}$ are negative. Following the same reasoning, it can be shown that the platform and social optimum are 0 . In region $\mathrm{F}$, the discriminant for $\hat{\hat{n}}_{2}^{h}$ is negative, so the social optimum is 0 . Moreover, $\hat{n}_{2}^{h}$ is negative and the coefficient of the term of degree 3 in $r^{h}$ is negative, hence $r^{h}$ is decreasing in the interval for $n_{2}^{h} \in\left[0, N_{2}\right]$. We illustrate this in Figure 2. Therefore, the platform optimum is also achieved at 0 . In region $\mathrm{G}$, both $\hat{n}_{2}^{h}$ and $\hat{\hat{n}}_{2}^{h}$ are negative.

\footnotetext{
${ }^{3}$ By simulation, we can show $v^{h}\left(\hat{\hat{n}}_{2}^{h}\right)>v^{h}(0)$ and $r^{h}\left(\hat{n}_{2}^{h}\right)>r^{h}(0)$ for region A. And we can show the same for region $\mathrm{B}$ by analysis. This is the only reason we differentiate regions $\mathrm{A}$ and $\mathrm{B}$.
} 


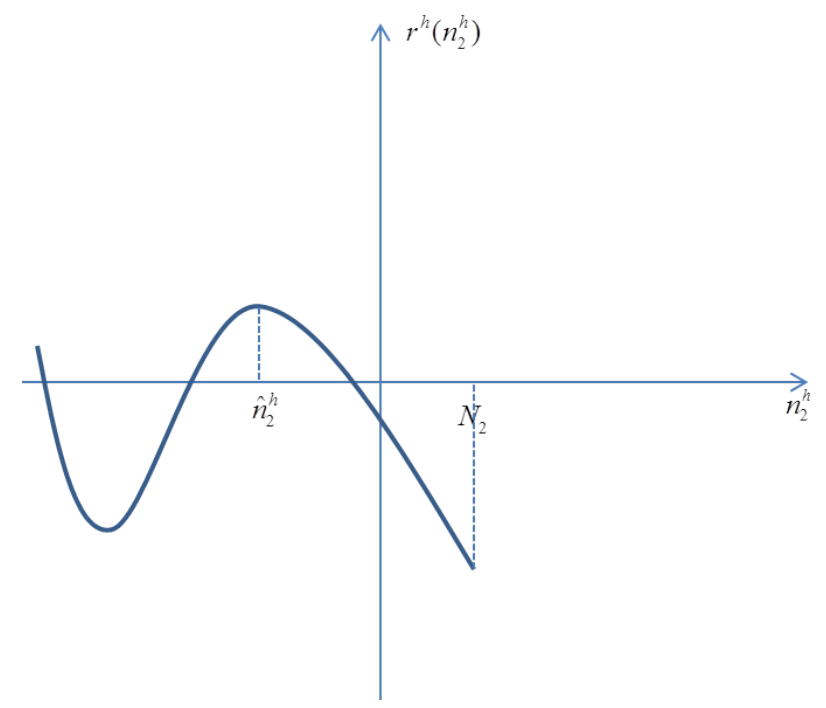

Figure 2: $r^{h}\left(n_{2}^{h}\right)$ for region $\mathrm{F}$

Hence, both $r^{h}$ and $v^{h}$ are decreasing in the interval $\left[0, N_{2}\right]$ and the platform and social optimum are 0 .

We summarize above discussions in the following proposition.

Proposition 4.1. In the competitive bottleneck model, taking quadratic within-group externalities among group 2 agents into account,

1. for sufficiently large $c_{2}$, e.g., regions $A$ and $B$, platform serves too few group 2 agents compared with the social optimum;

2. In region $C$, social optimum predicts 0 number of group 2 agents on the platform while the platform is still serving a positive number of group 2 agents for its own benefits;

3. for sufficiently small $c_{2}$, e.g., regions $D, E, F$ and $G$, both platform and social optimum predict 0 group 2 agents on the platform.

In Case 1, we get the same results as Armstrong (2006) that there are too few group 2 agents served on the platform. Note that in this case, both the platform and social optimum are positive numbers. For a large $b_{2}$, we need a small $c_{2}$ to sustain positive numbers of group 2 agents on the platform. Putting it differently, for a sufficiently large positive within-group externalities among group 2 agents, we need less group 1 agents on the platform to attract group 2 agents for a fixed constant marginal cost to serve group 2 agents. In Case 2, by adding within-group externalities, we get opposite result as Armstrong (2006), i.e., the platform still serves group 2 agents in the optimum but social optimum predicts 0 group 2 agents. For sufficiently small $c_{2}$, e.g., a higher fixed constant marginal cost to serve each group 2 agent or relatively less group 1 agents on the platform, both the platform and social optimum admit 0 group 2 agents. 


\section{References}

Armstrong, M. (2006). Competition in two-sided markets. RAND Journal of Economics 37(3), 668-691.

van Cayseele, P. and J. Reynaerts (2011). Complementary platforms. Review of Network Economics 10(1), 1-33.

Rochet, J.-C. and J. Tirole (2006). Two-sided markets: a progress report. RAND Journal of Economics 37, 645-667. 